\title{
A prática do Fisioterapeuta na Atenção Primária: Revisão integrativa
}

\author{
Rafaela Bresciani Marreto', Katia Terezinha Alves², Mara Quaglio \\ Chirelli ${ }^{2}$, Brunha Plassa ${ }^{1}$, Cassia Regina Fernandes Biffe ${ }^{2}$ e Silvia \\ Tonhom ${ }^{2}$
}

${ }^{1}$ Fisioterapeuta, Brasil | rafaela.marreto@gmail.com; bruna.plassa@hotmail.com.br | https://orcid.org/0000-0000-0000-0000; https://orcid.org/0000-0002-5424-6570

${ }^{2}$ Faculdade de Medicina de Marília, Brasil | katialvesrezende@gmail.com; marachirelli@gmail.com; c.r.biffe@gmail.com; siltonhom@gmail.com | https://orcid.org/00000002-9022-2680; http://orcid.org/0000-0002-7417-4439; https://orcid.org/0000-0002-84300400; http://orcid.org/0000-0001-7522-2861

\begin{abstract}
Resumo: Introdução: Historicamente, a fisioterapia é vista como profissão com uma prática essencialmente curativa e reabilitadora, sendo que sua inserção na atenção primária é relativamente nova. Entretanto, segundo as Diretrizes Curriculares Nacionais do Curso de Graduação em Fisioterapia, sabe-se que o fisioterapeuta possui competência para atuar no contexto da atenção primária de acordo com os princípios do SUS; Objetivos: Analisar as práticas, por meio da literatura, desenvolvidas pelos fisioterapeutas nos serviços de saúde da Atenção Primária à Saúde em relação ao cuidado individual; Métodos: Revisão integrativa da literatura, com busca realizada nas bases de dados LILACS, MEDLINE e BEDNF, utilizando os descritores "prática profissional", "fisioterapeuta", "fisioterapia", "atenção primária à saúde" e "estratégia saúde da família" nos idiomas português, inglês e espanhol. O período da busca compreendeu de 2016 a 2020; Resultados: A amostra final foi composta por dezenove artigos que foram analisados, sendo que, foram identificadas as áreas de competência à atenção à saúde, no âmbito do cuidado individual e coletivo, gestão a saúde e prática de pesquisa; Conclusões: Fisioterapeuta atuante na atenção primária realiza ações de caráter preventivo quanto curativo, contudo tem-se a necessidade de revisitar a formação na graduação.
\end{abstract}

Palavras-chave: Atenção Primária à Saúde; Fisioterapeuta; Prática Profissional.

\section{Physiotherapist Practice in Primary Care: An Integrative Review}

\begin{abstract}
Introduction Historically, physiotherapy has been regarded as profession with an essentially curative and rehabilitative practice and, its insertion in primary care is relatively new. However, according to the National Curricular Guidelines for the Undergraduate Physiotherapy Course, it is known that the physiotherapist has skills and abilities to act in the context of primary care in accordance with the principles of SUS; Goals Analyze the practices developed by physiotherapists in the health services of Primary Health Care in relation to individual care; Methods Integrative literature review, with a search conducted in the LILACS, MEDLINE and BEDNF databases, using the keywords "professional practice", "physiotherapist", "physiotherapy", "primary health care" and "family health strategy" in Portuguese, English and Spanish languages. The search period comprised the period 2016 to 2020.; Results The sample consisted of nineteen articles that were identified, and was identified as areas of competence, within the scope of individual and collective care, health management and research practice Conclusions. Physiotherapist working in primary care, performs preventive as well as curative actions, however there is a need to revisit training in undergraduate courses.
\end{abstract}

Keywords: Primary Health Care; Physiotherapist; Professional Practice.

\section{Introdução}

A fisioterapia é considerada como uma ocupação relativamente nova no Brasil, sendo regulamentada como profissão de nível superior somente em 1969 por meio do DecretoLei n 938, em que o art. 3 definiu que "é atividade exclusiva do fisioterapeuta executar métodos e técnicas fisioterápicas com a finalidade de restaurar, desenvolver e conservar a capacidade física do paciente" (Brasil, 1969). 
É evidente que esta definição apresenta-se ultrapassada no que se refere a práxis da fisioterapia e que, segundo Bispo (2010), este decreto remete ainda a uma restrição de sua atuação quanto aos níveis de atenção à saúde e que o mesmo não é mais compatível com a realidade do perfil epidemiológico e atual Sistema de Saúde do Brasil.

O fisioterapeuta teve sua inserção na Atenção Básica em 2003, a partir das discussões da $12^{\mathrm{a}}$ Conferência Nacional de Saúde (CNS), com aprovação da composição da equipe multiprofissional, incluindo o fisioterapeuta como apoio às equipes de Saúde da Família, de acordo com as necessidades de cada local. Em 2004, o Projeto de Lei oㅜ 4261/2004, inclui os profissionais de fisioterapia na Estratégia Saúde da Família (ESF) (Silva \& Ros, 2007).

Em 2008 foi implantado o Núcleo de Apoio à Saúde da Família (NASF), inserindo formalmente demais categorias profissionais na estratégia por meio de apoio matricial (AM). Aspectos estes que suscitaram muitos desafios, em especial, ao fisioterapeuta que tinha sua formação e ações mais voltadas para o tratamento de pessoas com alterações físico-funcionais em fases tardias dos agravos à saúde (Tavares, Costa, Oishi \& Driusso, 2018).

Nesse sentido, com a inserção do fisioterapeuta na APS, observa-se a necessidade de reconstruir sua prática, por meio de processos reflexivos, construindo estratégias para que estas vão ao encontro das ações de promoção à saúde de prevenção de doenças, recuperação e reabilitação esperadas nesse nível de atenção (Del Antonio, Tonhom \& Chirelli, 2019).

Nesse contexto de recente inserção do fisioterapeuta na atenção primária e diante de tantos desafios de reconstrução de sua prática, tem-se como pergunta de pesquisa: como está ocorrendo as práticas de fisioterapia acerca do cuidado individual, no contexto da atenção primária à saúde?

Assim, o objetivo dessa pesquisa foi analisar, por meio da literatura, as práticas desenvolvidas pelos fisioterapeutas nos serviços de saúde da Atenção Primária à Saúde em relação ao cuidado individual.

\section{Objetivo}

Analisar a prática do fisioterapeuta, em relação ao cuidado as necessidades individuais, nos serviços da atenção primária à saúde.

\section{Metodologia}

A revisão integrativa foi a estratégia utilizada para o desenvolvimento deste trabalho. É descrita por Broome (1993) apud Whitmore e Knalf (2005), como um método eficaz para definir conceitos, resumir conclusões e teorias de um determinado assunto e apresentar compreensões relevantes através de pesquisas já realizadas.

O desenvolvimento deste trabalho foi realizado em seis etapas para a elaboração da revisão integrativa, sendo elas: estabelecimento da questão da pesquisa, busca na literatura, categorização dos estudos, avaliação dos estudos encontrados, interpretação dos resultados, síntese do conhecimento ou apresentação da revisão (Mendes et al., 2008)

Inicialmente, foi elaborada a pergunta norteadora: "Como se dá a prática do fisioterapeuta na atenção básica/ primária da saúde no âmbito do cuidado individual?". Em seguida, foi realizado o levantamento bibliográfico por meio dos bancos de dados Literatura Latino Americana e do Caribe (LILACS), Sistema Online de Busca e Análise de Literatura Médica (MEDLINE) e Base de Dados de Enfermagem (BDENF) utilizando os descritores "prática profissional", "fisioterapeuta", "fisioterapia", "atenção primária à saúde" e estratégia saúde da família" nos idiomas português, inglês e espanhol. 
Para a seleção dos trabalhos, foram adotados como critérios de inclusão artigos de estudos primários que respondessem à pergunta de pesquisa, que foram publicados no período de 2016 a 2020, por se propor a reconhecer quais as práticas mais recentes na área pesquisada, nos idiomas português, inglês e espanhol e que estão disponíveis para acesso na integra.

Quanto aos critérios de exclusão, foram descartados artigos que não respondessem à pergunta da pesquisa, e que não estão disponíveis para acesso na integra. Também foram excluídas teses, dissertações e revisões, considerando-se que o foco está na identificação das práticas realizadas na ESF a partir de publicações revisadas pelos pares e estudos primários.

Nesta busca, foram inicialmente identificados um total de 80 estudos nas seguintes bases de dados: 49 na base de dados LILACS, 24 na base de dados MEDLINE e 7 na base de dados BDENF. Para determinar se os artigos eram elegíveis, realizou-se leitura exploratória de títulos e resumos. Nos casos em que as informações não foram suficientes, foi executada leitura dinâmica do artigo.

Após a seleção dos estudos, um total de 19, todos foram lidos integralmente e submetidos a análise em que foi realizada extração das informações. A Figura 1 apresenta as etapas realizadas.

A partir da leitura na integra dos artigos incluídos na revisão, classificou-se as informações de acordo com os autores, título do artigo, periódico, idioma, país e tipo de estudo. A seguir, fez-se uma leitura dos resultados e conclusões de cada artigo classificando-os em categorias, considerando as práticas realizadas pelo fisioterapeuta por área de competência: prática da fisioterapia no âmbito do cuidado às necessidades individuais, cuidado as necessidades coletivas, gestão e educação na saúde. Nesse artigo serão apresentados os resultados da área do cuidado as necessidades individuais.

O processo de seleção e codificação dos resultados foi realizado por uma pesquisadora e validado por uma outra, com doutorado, de forma independente.
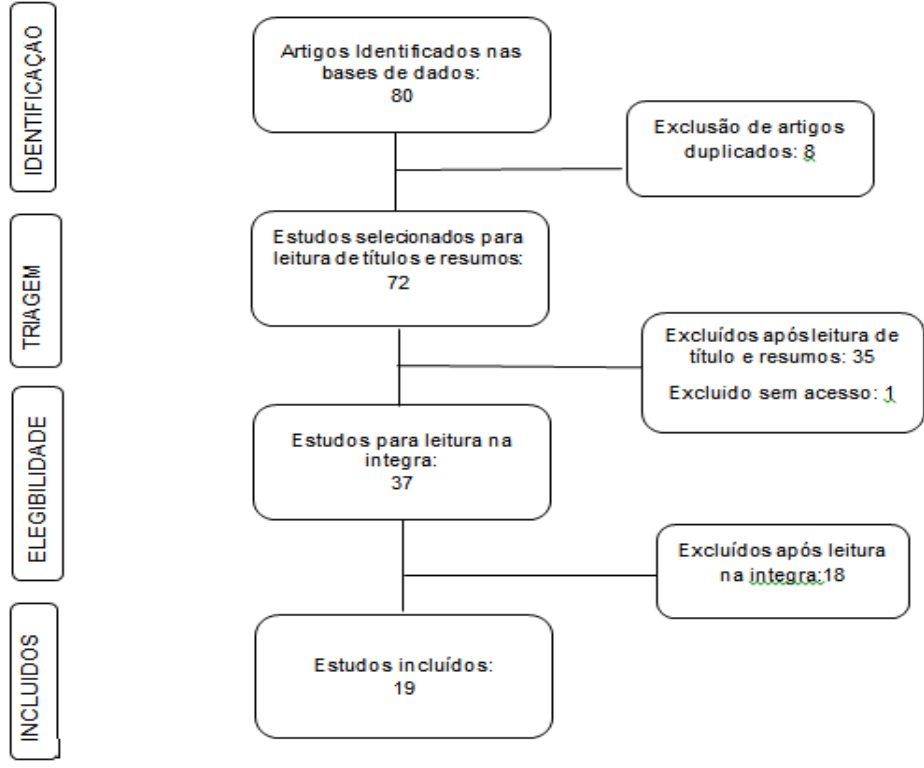

Fig.1. Fluxograma de seleção dos estudos primários, elaborado a partir da recomendação. PRISMA, 2021. 


\section{Resultados}

Em relação a como se dá a prática do fisioterapeuta na atenção primária a saúde evidencia-se três temáticas a saber, cuidado a necessidades individuais e coletivas e gestão em saúde. Nesse artigo, discorre-se sobre o cuidado as necessidades individuais.

\subsection{Cuidado as Necessidades Individuais}

Widerström, Rasmussen-Barr e Boström (2019) e Holm et al (2016) apresentam a atuação do fisioterapeuta na atenção primária na Suécia. Segundo Widerström, Rasmussen-Barr e Boström (2019) o cuidado pode ser ofertado por meio de clinicas públicas ou privadas e prestado de acordo com a necessidade de cada usuário e conhecimento do profissional, baseando-se na avaliação e quadro clinico. Utiliza-se como aplicação de métodos para redução de dor, prescrição de exercícios, educação e orientações sobre determinada patologia - lombalgia - e as vezes, associação de mais de um método de intervenção.

Enquanto isso, Holm et al (2016) também citam o profissional como referência na atenção primária, e que, em determinadas situações, como por exemplo, crianças e adolescentes com dores crônicas quando necessitam de cuidados, irão se consultar com um fisioterapeuta antes ou em conjunto com um clinico geral ou pediatra. Contudo, os autores não explicitam as estratégias ou intervenções realizadas neste âmbito.

O acesso para os serviços de fisioterapia na atenção primária na Irlanda assemelha-se ao da Suécia. Segundo Barrett, Hussey e Darker (2017), os serviços privados são disponíveis para indivíduos que pagam diretamente ou que possuem seguro médico privado. A atuação destes profissionais é pautada no modelo curativo em decorrência a alta demanda por parte de indivíduos que procuram tratamento devido alguma lesão ou condição médica.

No que se refere atenção à saúde no âmbito do cuidado individual na Holanda, Van Erp et al (2017) expressam a atuação do fisioterapeuta por meio de atendimentos individualizados, em que neste estudo, a assistência é direcionada a pessoas que possuem diagnóstico de dor lombar crônica não especifica e que não apresentam altos níveis de incapacidade funcional. As condutas normalmente realizadas por estes profissionais são, prescrição de exercícios, terapia manual e orientações.

Em relação à prática do fisioterapeuta no Reino Unido, Sarigiovannis e Cropper (2018) destacam que o mesmo é o profissional de referência para alguns usuários, sendo responsável por atendimentos individuais de casos complexos, assim como o planejamento destes. Além disso, tem-se a presença do assistente de fisioterapia que também pode atuar em clinicas para atender a população.

A oferta do cuidado individual em Andaluzia, Espanha, na atenção primária ocorre por meio de centros de saúde ou unidades móveis, segundo López-Liria et al (2016). Estas apresentam equipe composta por fisioterapeuta, fisiatra e terapeuta ocupacional. A assistência é ofertada de acordo com as necessidades individuais de cada usuário e com os objetivos de reabilitação. No estudo, o público alvo foram usuários com sequelas de AVE e alguns dos objetivos de tratamento eram: minimização de sequelas e prevenção de maiores complicações decorrentes do AVE, recuperação da capacidade funcional, estratégias para o usuário se adaptar ao seu quadro clinico, bem como o seu ambiente. Suporte para a família também foi ofertado com orientações e treinamento sobre a marcha do usuário, assim como informações sobre adaptações ergonômicas da residência.

Intervenções realizadas em domicílio, por equipe multiprofissional, visando a prevenção de complicações e a reabilitação de pacientes neurológicos também é evidenciada por Faria, Gonçalves e Silva (2016), que destacaram em seu estudo de caso - paciente com sequelas neurológicas e com outras comorbidades - a importância desta abordagem. 
A equipe foi constituída por fisioterapeuta, enfermeira e nutricionista e dentre as técnicas utilizadas para o atendimento do usuário, salientaram a abordagem da avaliação, programas de exercícios, ações preventivas relacionadas a úlceras por pressão (UPP) e orientações ao usuário e ao cuidador/familiar.

Faria, Araújo e Carvalho-Pinto (2016) também explicitam a assistência prestada a pacientes neurológicos, que neste caso, é direcionada a usuários que apresentam sequelas pós AVE. O cuidado ofertado pelo profissional inserido no NASF é realizado com maior frequência na residência do usuário por meio de visitas domiciliares e, com menor periocidade, na unidade. As ações se dão, principalmente, por meio de orientações e avaliações, seguida de tratamento e acompanhamento. $O$ cuidado integral a estes usuários, por meio de encaminhamentos para centros especializados de referência ou universidades e para outros profissionais do NASF, se faz presente, entretanto, foram descritas como pouco realizadas.

A prática de visitas domiciliares, principalmente a usuários que apresentam dificuldades ou impossibilidade de se deslocar a unidade, mediante requisição da equipe de referência é descrita por Barghini, Ferreti e Ferraz (2016) e Barghini, Ferreti e Ferraz (2017), sob a perspectiva dos gestores e equipe de referência e, dos próprios profissionais, respectivamente.

As visitas podem ser individuais ou compartilhadas e têm como propósito ofertar orientações para prevenir deformidades e lesões devido a ulceras de pressão e estimular a prática de exercícios. Ações de educação em saúde e de prevenção de doenças por meio de informações sobre estilo e hábitos de vida mais saudáveis durante consultas individuais também são citadas como práticas recorrentes. Além destas atividades, temse a execução de atendimentos voltados a oxigenoterapia e a auriculoterapia (Barghini, Ferreti \& Ferraz, 2016; Barghini, Ferreti \& Ferraz 2017).

A prática reabilitadora produzida pelo fisioterapeuta do NASF se faz presente nos estudos de Assis et al (2017) e Luna et al (2018). Ambos apresentam a predominância de atendimentos a pediatria na unidade de saúde, que assume papel de centro de referência devido à ausência deste serviço no território e a alta demanda. Este cenário faz com que atividades essenciais e preconizadas, como apoio matricial tecnopedagógico e ações de prevenção de agravos e promoção a saúde sejam pouco realizadas. Além disso, Luna et al (2018) expressam a inexistência da prática de visitas domiciliares, ao contrário de Assis et al (2017), que cita o apoio ofertado pelo fisioterapeuta residente junto ao NASF na realização desta atividade, seja em seu núcleo ou de forma compartilhada com outros profissionais, para usuários acamados ou impossibilitados de sair de sua residência.

De acordo com Souza e Santos (2017), Fernandes et al (2017) e Guedes et al (2016), a atenção a saúde neste cuidado dá-se por meio de visitas e atendimentos domiciliares e na USF. Para Fernandes et al (2017) há predomínio de consultas individuais visando a reabilitação, quando comparadas a outras atividades realizadas na rotina do fisioterapeuta e que alguns profissionais realizam a clínica ampliada (CA) e projeto terapêutico singular (PTS). A falta de familiaridade e a formação deficitária dos profissionais dificultam a operacionalização do cuidado nesta perspectiva. Enquanto isso, Souza e Santos (2017) expressam a visita domiciliar como atividade mais executada pelo profissional, além da realização de PTS para o manejo do cuidado do usuário.

Guedes et al (2016), não especificam em seu relato de experiencia quais destas atividades são realizadas com maior frequência, contudo, os autores destacam que as visitas e atendimentos domiciliares são de grande valia, tanto para os profissionais quanto aos usuários, e devem ser realizadas independente se os indivíduos apresentam alguma patologia, pois este tipo de assistência apresenta impacto positivo nas condições de saúde.

A atuação do fisioterapeuta junto a equipe multiprofissional por meio de programas de residência é revelada por Araújo et al (2018), Sales (2016) e Silva et al (2016). 
O cuidado individual ofertado pelo fisioterapeuta residente é apresentado por Sales (2016) por meio de visitas ou atendimentos domiciliares e na USF. A construção deste cuidado é realizada mediante a caracterização, necessidades e demandas do território e desenvolvida junto a equipe de saúde respeitando determinados critérios de elegibilidade, como por exemplo, presença de doenças crônico degenerativas, restrição ao leito, ausência de acompanhamento prévio ou atendimento em outros serviços especializados, dificuldade de deslocamento ou de compreensão da família quanto as orientações fornecidas pelo profissional. $\mathrm{O}$ autor cita que, em relação aos acamados, tem-se a prática de visitas com fornecimento de orientações aos familiares, preconizadas para cada quadro clinico. Já em relação ao atendimento individualizado, houve prevalência de consultas de crianças com atraso no desenvolvimento neuropsicomotor, usuários com queixas ortopédicas, pós-cirúrgico e pacientes que apresentavam diagnóstico de doenças crônico degenerativas.

A inserção do fisioterapeuta residente junto a uma ampla variedade de profissionais como cirurgião-dentista, assistente social, enfermeira, nutricionista, psicólogo, farmacêutico e fonoaudiólogo inseridos na atenção primária é retratada por Araújo et al (2018). A estratégia apresentada é a consulta coletiva e compartilhada com enfoque na CA na puericultura. Segundo os autores, o fisioterapeuta pode realizar avaliação da criança com a finalidade de identificar possíveis patologias e alterações do desenvolvimento neuropsicomotor (DNPM), assim como fornecer orientações ao responsável sobre estímulos para o desenvolvimento da criança e, caso haja necessidade, referenciar para serviço especializado. Além disso, pode ofertar estratégias de educação em saúde para prevenção de acidentes domésticos.

Por último, Silva et al (2016), exploram a atuação do residente inserido em uma estratégia intitulada "Estratégia Vigilância à Dor Crônica de Origem Osteomioarticular", em que o profissional exerce papel de avaliar o indivíduo e identificar as necessidades do usuário, seguindo critérios para cada caso. Durantes estes atendimentos, tem-se a prática de realizar orientações sobre determinada patologia que o indivíduo apresente, sobre hábitos saudáveis, estimulo a adesão da prática de exercícios físicos na rotina e técnicas para analgesia e melhora do quadro clinico e qualidade de vida. Além do mais, durante estas consultas, caso haja necessidade, o fisioterapeuta pode referenciar estes indivíduos para grupos, centros ou a outros profissionais.

\section{Discussão}

O fisioterapeuta apresenta crescimento principalmente na atenção hospitalar e um leve acréscimo na APS, contudo quando observadas as necessidades por estes profissionais, tem-se desigualdade quanto ao acesso a estes pela população (Rodes et al, 2017).

Nesse sentido, Neto e Aguiar (2018), indicam que políticas de inclusão do fisioterapeuta nos NASFs foram implementadas, mas as atribuições desse profissional ainda não foram totalmente estabelecidas, dificultando o cuidado à população e a formação profissional.

Rodes et al (2017), analisando o discurso dos fisioterapeutas formados entre $1981 \mathrm{e}$ 2007, explicita que houve capacitação para APS e início do trabalho do NASF. Quanto ao uso das ferramentas como CA, PTS, AM, levantam os desafios enfrentados pelos profissionais como regime de trabalho com carga horaria reduzida, comunicação entre membros da equipe e falta de compreensão de determinados conceitos, o que torna difícil a utilização destes recursos na prática.

Segundo Neto e Aguiar (2018), ainda existe uma relação entre prática e formação profissional, a inovação na formação não muda a prática e mudanças nessa não determinam novos rumos para a formação. O mundo do trabalho em APS ainda é escasso para fisioterapeutas, mesmo que esse profissional seja essencial na atenção em unidades do SUS. Portanto, considera-se que a graduação pode arquitetar para que os egressos assumam uma atitude ativa quanto a identificação e atenção às necessidades de saúde da população, fortalecendo a ESF. 
Melo et al (2020), que abordam a atenção integral a saúde da criança realizada pelos profissionais inseridos no NASF, em especial o trabalho dos fisioterapeutas, evidenciam a alta demanda e filas de espera pelos serviços especializados e centros de reabilitação e que, por meio dos grupos desenvolvidos pelo NASF no território, tem-se a possibilidade de realizar ações que visam o estimulo do desenvolvimento, com atuação transdisciplinar destes profissionais. Concluem que práticas multiprofissionais e interdisciplinares são essenciais na promoção no cuidado em saúde, sendo os nasfianos capazes de realizar intervenções de forma precoce.

Os profissionais matriciadores, dentre eles, o fisioterapeuta, podem oscilar, conforme propósito individual ou grupal, desviando-se da ação clínica especializada, atuando mais em reuniões como suporte técnico pedagógico às equipes, grupos, ações educativas e preventivas; ou desviando-se para seus núcleos de competência e limitando-se a promover cuidados especializados. Nessa última tendência, elabora-se uma agenda que rapidamente se finda, sem negociação ou suporte às equipes em dúvidas/urgências e sem construção coletiva de PTS complexos. (Tesser, 2017).

Para Neto e Aguiar (2018), os conhecimentos se modificam e os processos de trabalho são distintos em diferentes contextos de prática. A diversidade de dinâmicas de funcionamento dos NASFs com distintas realidades locais pode dificultar a formulação de uma estratégia de ensino pelos cursos de Fisioterapia. Como a graduação não esgota a formação, cabe capacitar o aluno na perspectiva da educação continuada e permanente.

\section{Conclusão}

A abordagem qualitativa da investigação permite afirmar que a prática do profissional na atenção primária foca-se em atividades relacionadas a atendimentos na unidade de saúde e no domicilio do usuário, sendo que ambas podem ser promovidas de forma individual ou em parceria com outros profissionais.

Estas atividades contemplam ações relacionadas a prevenção de agravos, promoção $e$ educação a saúde e reabilitação, contudo, cabe ressaltar o evidente predomínio e ênfase de consultas configuradas na lógica curativo reabilitadora.

Tendo em vista que pretende-se promover o cuidado na perspectiva da integralidade, há necessidade de revisitar a formação na graduação, bem como investir na educação continuada e permanente.

\section{Referências}

Araújo, D. C. de, Lucena, E. E. de S., Tavares, T. R. P., Araújo, T. B. de, Araújo, C. M. de, Costa, B. M. B. da, Medeiros, B. G. de, Gomes, B. C. do N., Vale, T. R. F., Dantas, L. R. de O., \& Medeiros Filho, J. S. de A. (2019). Promoção de Saúde Bucal nas Consultas de Crescimento e Desenvolvimento na Atenção Primária: Um relato de colaboração interprofissional. Revista Ciência Plural, 4(2), 87-101.

Assis, J. C. L., Arruda, G. M. M. S., Bezerra, M. I. C., \& de Vasconcelos, T. B. (2016). A vivência de um fisioterapeuta em uma Residência Multiprofissional: desafios e possibilidades. Revista de APS, 20(2).

Barrett, E. M., Hussey, J., \& Darker, C. D. (2017). Feasibility of a physical activity pathway for Irish primary care physiotherapy services. Physiotherapy, 103(1), 106-112. https://doi.org/10.1016/j.physio.2016.02.001

Braghini, C. C., Ferretti, F., \& Ferraz, L. (2016). Atuação do fisioterapeuta no NASF: percepção de coordenadores e equipe. Fisioterapia em Movimento , 29 (4), 767-776. https://dx.doi.org/10.1590/1980-5918.029.004.ao13

Braghini, C. C. , Ferretti, F., \& Ferraz, L. (2017). O papel do fisioterapeuta no contexto dos centros de apoio à saúde da família. Fisioterapia em Movimento , 30 (4), 703-713. https://dx.doi.org/10.1590/1980-5918.030.004.ao05 
Bispo Júnior, J. P. (2010). Fisioterapia e saúde coletiva: desafios e novas responsabilidades profissionais. Ciência \& Saúde Coletiva, 15(Suppl. 1), 1627-1636. https://doi.org/10.1590/S1413-81232010000700074

Brasil. (1969) Decreto-Lei 938. Provê sobre as profissões de Fisioterapeuta e Terapeuta Ocupacional, e dá outras providências. Diário Oficial da União, 14 out.

Brasil. (2012). Política nacional de atenção básica.

Del Antonio, A. C. F. T., Tonhom, S. F. R. \& Chirelli, M. Q. (2019). Desenvolvimento curricular e formação do fisioterapeuta: desafios do cuidado na Atenção Primária à Saúde. Indagatio Didactica, 11 (3), 131-147.

Faria, C. D. C. M., Araújo, D. C., \& Carvalho-Pinto, B. P. B. (2017). Assistência prestada por fisioterapeutas desde a atenção primária à saúde aos pacientes após AVC. Fisioterapia em Movimento , 30 (3), 527-536. https://dx.doi.org/10.1590/1980-5918.030.003.ao11

Faria, L., Gonçalves, M. do C. P., \& Silva, E. B. (2016). Fisioterapia preventiva e humanização do cuidado no tratamento do paciente neurológico, acamado, domiciliar. Fisioterapia em Movimento , 29 (1), 13-22. https://doi.org/10.1590/0103-5150.029.001.AO01

Fernandes, J. M, Rios, T. A., Sanches, V. S. \& Santos, M. L. de M. dos. (2016). Ferramentas e práticas do NASF em saúde do fisioterapeuta. Fisioterapia em Movimento , 29 (4), 741-750. https://doi.org/10.1590/1980-5918.029.004.ao10

Guedes, M. B. O. , Lima Filho, B. F, Pinheiro, H. V., Lopes, J.M. \& Souza, C. G. Atenção fisioterapêutica em duas unidades básicas de saúde em um município do Rio Grande do Norte: Um contato humanizado. Rev. APS. Vol. 19 n. 1, p. 150-153. Janeiro 2016.

Holm, S., Ljungman, G., Åsenlöf, P., Linton, S. J., \& Söderlund, A. (2016). Treating youth in pain: Comparing tailored behavioural medicine treatment provided by physical therapists in primary care with physical exercises. European journal of pain (London, England), 20(4), 626-638. https://doi.org/10.1002/ejp.780

López-Liria, R., Vega-Ramírez, F. A., Rocamora-Pérez, P., Aguilar-Parra, J. M., \& Padilla-Góngora, D. (2016). Comparison of Two Post-Stroke Rehabilitation Programs: A Follow-Up Study among Primary versus Specialized Health Care. PloS one, 11(11), e0166242. https://doi.org/10.1371/journal.pone.0166242

Luna, M., Lucena, P., Farias, A., \& Melo, V. (2018). O acompanhamento fisioterapêutico de crianças com sequelas de paralisia cerebral atendidas no NASF do município de alagoa nova. Revista de Pesquisa: Cuidado é Fundamental Online, 10 (Especial), 70-73. doi:http://dx.doi.org/10.9789/2175-5361.2018.v10iEspecial.70-73

Mélo, T. R. , Lucchesi, V. de O., Ribeiro Junior, E. J. F., \& Signorelli, M. C.. (2020). Caracterização do desenvolvimento neuropsicomotor e de linguagem de crianças atendidas por grupos no Núcleo Ampliado de Saúde da Família: uma abordagem interprofissional. Revista CEFAC, 22(3), e14919

Mendes, K. D. S., Silveira, R. C. de C. P., \& Galvão, C. M. (2008). Revisão integrativa: método de pesquisa para a incorporação de evidências na saúde e na enfermagem. Texto \& Contexto Enfermagem, 17(4), 758-764. https://doi.org/10.1590/S0104-07072008000400018

Rangel Neto, N. C., \& Aguiar, A. C. de. (2018). A atenção primária à saúde nos cursos de graduação em fisioterapia no municipio do Rio de Janeiro. Trabalho, Educação e Saúde, 16(3), 1403-1420. https://dx.doi.org/10.1590/1981-7746-sol00165

Rodes, C. H, Kurebayashi, R., Kondo, V. E., Luft, V. D, Góes, Â. B. de, \& Schmitt, A. C. Bo. (2017). O acesso e o fazer da reabilitação na Atenção Primária à Saúde. Fisioterapia e Pesquisa, 24(1), 74-82

Sales, R. D. C. (2016) O papel do fisioterapeuta residente multiprofissional em saúde da família: um relato de experiência. Rev APS 19(3):500-4.

Sarigiovannis, P., \& Cropper, S. (2018). An audit of the utilization of physiotherapy assistants in the musculoskeletal outpatients setting within a primary care physiotherapy service. Musculoskeletal care, 16(3), 405-408. https://doi.org/10.1002/msc.1238

Silva, D. J. \& Ros, M. A. (2007). Inserção de profissionais de fisioterapia na equipe de saúde da família e Sistema Único de Saúde: desafios na formação. Revista Ciências e Saúde Coletiva, 2 (6), 73-81. 
Silva, F. E. D., Dantas, F. R. P., Macena, R. H. M., \& Vasconcelos, T. B. (2016). Processo de implantação da estratégia vigilância à dor crônica osteomioarticular na atenção básica. Estudo de Caso. Revista Dor, 17(1), 69-72. https://dx.doi.org/10.5935/1806-0013.20160017

Starfield, B. (2006). Atenção primária: equilíbrio entre necessidades de saúde, serviços e tecnologia.

Souza, M. de O., \& Santos, K. O. B. dos. (2017). Atuação do fisioterapeuta no Centro de Apoio à Saúde da Família. Fisioterapia em Movimento , 30 (2), 237-246. https://dx.doi.org/10.1590/1980-5918.030.002.ao04

Tavares, L. R. C., Costa, J. L. R., Oishi, J. \& Driusso, P. (2018). Inserção da fisioterapia na atenção primária à saúde: análise do cadastro nacional de estabelecimentos de saúde em 2010. Fisioter Pesqui. 25(1):9-19. DOI: 10.1590/1809-2950/15774625012018

Van Erp, R., Huijnen, I., Köke, A., Abbink, F. E., den Hollander, M., \& Smeets, R. (2017). Development and content of the biopsychosocial primary care intervention 'Back on Track' for a subgroup of people with chronic low back pain. Physiotherapy, 103(2), 160-166. https://doi.org/10.1016/j.physio.2016.04.004

Vendruscolo, C., Metelski, F. K., Maffissoni, A. L., Tesser, C. D., \& Trindade, L. de L. (2020). Características e atuação dos profissionais dos Núcleos Ampliados de Saúde da Família e Atenção Básica. Revista da Escola de Enfermagem da USP, 54, e03554. Epub March 30, 2020.https://doi.org/10.1590/s1980-220x2018033003554

Widerström, B., Rasmussen-Barr, E., \& Boström, C. (2019). Aspects influencing clinical reasoning and decision-making when matching treatment to patients with low back pain in primary healthcare. Musculoskeletal science \& practice, 41, 6-14. https://doi.org/10.1016/j.msksp.2019.02.003 